\title{
Detecção de leucócitos em imagens de vídeo de microscopia intravital usando a técnica de congruência de fase
}

\section{Detection of leukocytes in intravital microscopy video images using the phase congruency technique}

\author{
Kathiani Elisa de Souza 1, 2 \\ Bruno C. Gregório da Silva ${ }^{1,3}$ \\ Juliana Carvalho-Tavares 4,5 \\ Ricardo J. Ferrari ${ }^{1,6}$
}

Data de submissão: 30/03/2016, Data de aceite: 20/06/2016

\begin{abstract}
Resumo: A quantificação do número de leucócitos rolantes e aderidos presentes na microcirculação de pequenos animais é uma tarefa importante para elucidar os mecanismos de inflamações e avaliar os efeitos terapêuticos de novos fármacos. Em geral, a contagem de leucócitos é realizada de maneira visual por um observador (técnico laboratorial ou especialista) usando uma sequência de imagens de microscopia intravital (MI). Entretanto, tal tarefa é demorada e suscetível a erros, devido a fadiga visual do observador e a variabilidades inter e intra observadores. Neste trabalho uma nova técnica computacional é proposta para a detecção automática de leucócitos em vídeos de MI. A técnica usa uma medida de blobness, calculada a partir da análise dos autovalores de matrizes locais de momentos de segunda ordem da medida de congruência de fase, para realçar os leucócitos nas imagens. A detecção dos leucócitos é alcançada pela busca de máximos locais no mapa de medidas de blobness. Usando um conjunto de quadros com os centroides dos leucócitos manualmente marcados, os resultados da técnica proposta foram avaliados usando os valores das métricas medidaF1 e áreas sob as curvas precisão-revocação (AUCPRs) calculadas para cada quadro do vídeo. Uma comparação com a técnica de casamento de padrões também foi realizada. Os resultados obtidos para a técnica proposta $\overline{(\text { medida }-\mathrm{F} 1}=0,791$, $\overline{\mathrm{AUCPR}}=0,776$ ) foram superiores em comparação com a técnica de casamento de padrões $(\overline{\text { medida }-\mathrm{F} 1}=0,746, \overline{\mathrm{AUCPR}}=0,670)$.
\end{abstract}

\footnotetext{
${ }^{1}$ Departamento de Computação, Universidade Federal de São Carlos - São Carlos, SP, Brasil. Caixa Postal 676.

2 kkathiani.souza@dc.ufscar.br\}

3 \{ bruno.silvaedc.ufscar.br\}

${ }^{4}$ Departamento de Fisiologia e Biofísica, Universidade Federal de Minas Gerais - Belo Horizonte, MG, Brasil. Caixa Postal 486.

${ }^{5}$ \{julianateicb.ufmg.br\}

${ }^{6}$ \{rferrariedc.ufscar.br\}
} 
Detecção de leucócitos em imagens de vídeo de microscopia intravital usando a técnica de congruência de fase

Palavras-chave: detecção de leucócitos, congruência de fase, microscopia intravital, microcirculação, resposta inflamatória

\begin{abstract}
Quantification of the number of rolling and adherent leukocytes present in the microcirculation of small animals is an important task to elucidate inflammation mechanisms and to assess therapeutic effects of new drugs. In general, leukocyte counting is done visually by an observer (laboratory technician or specialist) using an intravital microscopy image sequence. However, such task is time-consuming and susceptible to errors, due to visual fatigue of the observer and to inter and intra-observer variabilities. In this work a new computational technique is proposed for the automatic detection of leukocytes in intravital video microscopy. The technique uses a blobness measure, calculated from eigenanalysis of local matrices of second-order moments of the phase congruency measure, to enhance leukocytes in the images. Detection of leukocytes is achieved by searching for local maxima in the map of blobness measures. Using a set of frames with the leukocyte centroids manually annotated, the results were evaluated using the F1-measure and the area under the precision-recall curves (AUCPRs). The results for the proposed technique ( $\overline{\mathrm{F} 1-\text { measure }}=0,791$, $\overline{\mathrm{AUCPR}}=0,776$ ) were superior in comparison with the template matching technique $(\overline{\mathrm{F} 1-\text { measure }}=0,746, \overline{\mathrm{AUCPR}}=0,670)$.
\end{abstract}

Keywords: detection of leukocytes, phase congruency, intravital microscopy, microcirculation, inflammatory response

\title{
1 Introdução
}

O envolvimento da microcirculação no insulto inflamatório é compreendido por complexas interações e mecanismos biológicos de doenças associadas a diversas condições inflamatórias [25, 28]. Apesar da existência de um diversificado conjunto de sistemas experimentais in vitro para a análise da morfologia e de funções moleculares de células imunológicas, a maneira mais eficaz (quando não única) para medir tais atributos é por meio de técnicas de imageamento in vivo.

Por possuir características desejáveis, como grande profundidade focal e alta resolução espacial e temporal, a microscopia intravital (MI) [18] tornou-se uma ferramenta essencial para o estudo de sistemas biológicos in vivo, proporcionando a visualização do comportamento de células do sistema imunológico em órgãos linfoides e em tecidos periféricos. Com o seu uso é possível, por exemplo, avaliar o tráfego celular e mensurar o número de células presentes na microcirculação de pequenos animais em experimentos in vivo e nos mais diferentes contextos inflamatórios, facilitando, assim, a compreensão de mecanismos imunológicos de doenças e auxiliando o desenvolvimento de novos fármacos [24, 1]. 
Detecção de leucócitos em imagens de vídeo de microscopia intravital usando a técnica de congruência de fase

Em geral, a contagem de leucócitos é realizada por um observador (técnico laboratorial ou pesquisador) experiente que observa as células diretamente nos quadros dos vídeos de MI. Entretanto, esse processo visual, além de demorado, é propenso a erros devido à fadiga visual do observador e à variabilidade inter- e intra-observadores, os quais podem afetar diretamente os resultados da contagem [5, 22]. Assim, o estudo e o desenvolvimento de técnicas computacionais automáticas para a detecção de leucócitos é de grande importância para auxiliar na tarefa de contagem de células e também para aumentar a confiabilidade dos resultados de experimentos realizados pela MI [4].

Algumas abordagens automáticas e semiautomáticas para a detecção e o rastreamento de leucócitos em imagens de MI foram propostas na literatura. Egmont-Petersen et al. [6], por exemplo, propuseram o uso de redes neurais artificiais (RNAs) treinadas com imagens de leucócitos sintéticos, gerados a partir de um modelo estocástico, e com imagens de leucócitos reais, manualmente selecionados dos quadros do vídeo. Ao todo, foram utilizadas 12 RNAs, sendo que 6 foram treinadas com imagens de leucócitos sintéticos e 6 com leucócitos reais. Segundo os autores, o melhor desempenho foi alcançado por uma RNA com 9 nós e treinada com imagens de leucócitos sintéticos, a qual apresentou um valor de área sob a curva ROC $18 \%$ maior que a melhor RNA treinada com imagens de leucócitos reais.

Ray et al. [26] apresentaram um modelo de contornos ativos cujo funcional de energia foi composto pelos seguintes componentes: (i) energia interna, usando medidas de rigidez e elasticidade do contorno, (ii) energia externa, baseada na técnica Gradient Vector Flow, e (iii) energia de restrição, composta por informações de tamanho, forma, posição e número de pontos do contorno discretizado dos leucócitos. O modelo proposto foi comparado com métodos baseados em correlação cruzada e rastreadores de centroides e, de acordo com os autores, o método de contornos ativos obteve o melhor desempenho para a métrica da raiz quadrada do erro médio quadrático e também para o percentual de quadros rastreados.

Dong et al. [5] usaram também um modelo de contornos ativos para a detecção automática de leucócitos. O modelo proposto utilizou como representação paramétrica a técnica $B$-Spline [19] e como energia de restrição uma medida denominada Gradient Inverse Coefficient of Variation. Essa medida pode ser entendida como a razão entre a média e o desvio padrão das derivadas direcionais, calculadas sobre o contorno da célula na imagem. A identificação inicial das células foi realizada pela técnica de casamento de padrões. Após essa etapa, o modelo de contornos ativos foi aplicado para o refinamento das bordas estimadas. Como resultado na detecção dos leucócitos, o método apresentou um valor de acurácia de $78,6 \%$ com taxa de falsos positivos (FPs) de 13,1\%.

Um dos principais problemas que pode comprometer técnicas computacionais automáticas de detecção de leucócitos é a variação de contraste que os leucócitos apresentam ao longo dos quadros do vídeo. Esse efeito, conhecido como Photobleaching [2], causa a redução e, em último caso, a eliminação da emissão de fluorescência do leucócito ao longo do 
Detecção de leucócitos em imagens de vídeo de microscopia intravital usando a técnica de congruência de fase

tempo, fazendo com que este perca o contraste e se torne mais difícil de ser identificado nas imagens [30]. Em geral, técnicas baseadas na intensidade dos níveis de cinza da imagem não são suficientemente robustas para lidar com o efeito Photobleaching.

Neste trabalho, uma nova abordagem baseada na técnica de Congruência de Fase (Phase Congruency - PC) [12] é proposta para a detecção automática de leucócitos em imagens de vídeo de MI. A técnica de PC, que é conhecida por ser robusta às variações de contraste e iluminação na imagem, foi utilizada para o cálculo de uma medida de blobness que é usada para realçar estruturas na forma de bolha nas imagens de MI, as quais corresponderiam aos leucócitos.

Este artigo está organizado da seguinte maneira. A Seção 1 apresenta a motivação para a realização desta pesquisa e os principais trabalhos publicados na literatura que são diretamente relacionados a esta pesquisa. A descrição da metodologia empregada no desenvolvimento da técnica proposta é descrita na Seção 2. Resultados e discussões são apresentados na Seção 3 , seguido das conclusões na Seção 4

\section{Metodologia}

As principais etapas de processamento que compõem a técnica desenvolvida para a detecção automática de leucócitos em vídeos de MI são apresentadas na Figura 1 e descritas em detalhes nas subseções que seguem.

\subsection{Pré-processamento}

Dois efeitos indesejáveis frequentemente presentes nas imagens de vídeo de MI são o borramento e o desalinhamento de quadros do vídeo, causados, principalmente, por movimentos de respiração e batimentos cardíacos do animal de teste. Para minimizar tais efeitos e potencializar o sucesso da técnica de detecção de leucócitos, as imagens foram préprocessadas usando a seguinte sequência de etapas, na ordem enumerada:

1. Os quadros com excessivo borramento ou distorções foram identificados pela técnica proposta em Ferrari et al. [7] e removidos do vídeo. A remoção dos quadros só foi possível pois, para a aquisição do vídeo, foi utilizada uma alta taxa de amostragem. Assim, a continuidade do movimento dos leucócitos pôde ser mantida, mesmo após a eliminação dos quadros degradados.

2. O filtro Bilateral [29], que possui a propriedade de preservar bordas enquanto realiza o borramento da imagem, foi utilizado para reduzir o nível de ruído e melhorar a qualidade das imagens. Os parâmetros do filtro, que são o tamanho da janela de vizinhança 
Detecção de leucócitos em imagens de vídeo de microscopia intravital usando a técnica de congruência de fase

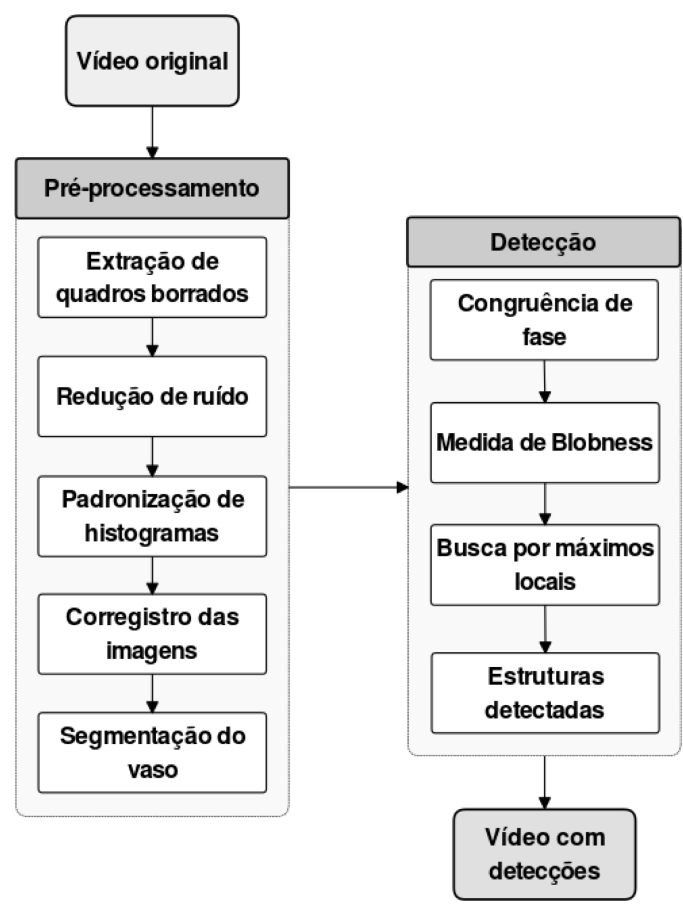

Figura 1: Principais etapas da técnica proposta para a detecção automática de leucócitos.

do pixel ( $d=9$ pixels) e as ponderações de intensidade $\left(\sigma_{r}=10\right)$ e espacial $\left(\sigma_{d}=10\right)$, foram experimentalmente ajustados para prover o melhor equilíbrio entre a redução de ruído e o borramento introduzido na imagem.

3. Para minimizar o efeito Photobleaching sobre as imagens do vídeo e melhorar os resultados da estabilização do vídeo pela técnica de corregistro das imagens, a técnica de casamento de histogramas proposta em Nyul et al. [23] foi aplicada a cada par de quadros consecutivos do vídeo.

4. O vídeo foi estabilizado usando a técnica proposta em Gregório da Silva et al. [11], que é baseada no corregistro de imagens, usando transformações afins e deformáveis, aplicado a cada par de quadros consecutivos do vídeo.

5. Uma máscara binária da região efetiva do vaso sanguíneo foi obtida usando o método proposto por Sato et al. [27]. Esse método calcula a variância temporal de cada pixel ao longo dos quadros do vídeo. Uma operação morfológica de abertura (elemento 
Detecção de leucócitos em imagens de vídeo de microscopia intravital usando a técnica de congruência de fase

estruturante de forma elíptica de tamanho $15 \times 15$ pixels) foi utilizada para eliminar pequenas vênulas na imagem. Finalmente, a máscara binária da região de fluxo (região de interesse) sanguíneo foi determinada como a estrutura de maior área na imagem.

Maiores detalhes a respeito das etapas de pré-processamento descritas acima podem ser obtidas nas referências [10, 11].

\subsection{Técnicas utilizadas na detecção dos leucócitos}

A técnica de Congruência de Fase (Phase Congruency - PC), proposta inicialmente por Morrone e Owens [21] e, mais tarde, aprimorada por Kovesi [14, 13, 12], permite o realce de características visuais importantes, do ponto de vista de modelos de percepção visual humana [20], em uma imagem. Diferentemente dos métodos baseados em informações de gradiente da imagem [17, 3, 16], a medida de PC (definida logo abaixo) é robusta a variações de contraste e iluminação das imagens.

Neste estudo, a técnica de PC foi utilizada para o cálculo de uma medida de realce de estruturas em forma de bolha, denominada medida de blobness, e empregada para a detecção de leucócitos presentes nos quadros de vídeo de MI.

2.2.1 Congruência de Fase Com base no modelo de energia local de Morrone e Owens [21], Kovesi [13] definiu a medida de PC para imagens bidimensionais como:

$$
\mathrm{PC}_{2 \mathrm{D}}(\mathbf{x})=\frac{\sum_{s=1}^{S} \sum_{n=1}^{N} W_{s, n}(\mathbf{x})\left\lfloor A_{s, n}(\mathbf{x}) \Delta \Phi_{s, n}(\mathbf{x})-T_{n}\right\rfloor}{\sum_{s=1}^{S} \sum_{n=1}^{N} A_{s, n}(\mathbf{x})+\epsilon},
$$

em que, \lfloor\rfloor indica que a quantidade contida dentro dos delimitadores é igual a ela mesma, se $\mathrm{o}$ valor for positivo, ou zero se o valor for negativo; $\mathbf{x}=(x, y)$ indica a localização espacial e $A_{s, n}$ é a energia da imagem na localização x calculada usando um filtro de log-Gabor 2D com escala $s$ e orientação $n$. $T_{n}$ é um limiar automaticamente estimado que representa a parcela da energia que se espera corresponder ao ruído [13]. O termo

$$
W_{s, n}(\mathbf{x})=\frac{1}{1+\exp \left\{\gamma\left[c-\frac{1}{S}\left(\frac{\sum_{s} \sum_{n} A_{s, n}(\mathbf{x})}{A_{\max }(\mathbf{x})+\epsilon}\right)\right]\right\}},
$$

é a função sigmoide de ponderação usada para corrigir a medida de PC de distribuições de frequências que são particularmente estreitas, visto que um ponto de congruência de fase só é significante se ocorrer em uma ampla faixa de frequências. Os parâmetros $\gamma \mathrm{e} c$ representam, respectivamente, o fator de ganho e o valor de corte da sigmoide e $\epsilon=0.0001$ é uma constante de valor pequeno para evitar divisão por zero. 
Detecção de leucócitos em imagens de vídeo de microscopia intravital usando a técnica de congruência de fase

O termo $\Delta \Phi$ na Equação (1) é uma expressão usada para melhorar a localização da resposta da medida de $\mathrm{PC}$, e é calculado como

$$
\begin{aligned}
\Delta \Phi_{s, n}(\mathbf{x})= & \cos \left(\phi_{s, n}(\mathbf{x})-\bar{\phi}_{n}(\mathbf{x})\right)- \\
& \left|\sin \left(\phi_{s, n}(\mathbf{x})-\bar{\phi}_{n}(\mathbf{x})\right)\right|,
\end{aligned}
$$

sendo que $\bar{\phi}_{n}(\mathbf{x})$ é o valor da fase média na posição $\mathbf{x}$.

2.2.2 Banco de filtros de log-Gabor 2D O banco de filtros de log-Gabor 2D é projetado no domínio da frequência e as funções de transferência dos filtros que o compõem são calculadas como [14]:

$$
G(\omega, \theta)_{\mid \omega_{s}, \theta_{n}, \sigma_{\beta}}=\exp \left\{-0.5\left[\frac{\log ^{2}\left(\frac{\omega}{\omega_{s}}\right)}{\log ^{2}\left(\sigma_{\beta}\right)}\right]\right\} \times\left[\frac{\cos (d \theta)+1}{2}\right],
$$

com

$$
d \theta=\min \left(\left|\arctan \left(\sin \left(\theta-\theta_{n}\right), \cos \left(\theta-\theta_{n}\right)\right)\right| \times \frac{N}{2}, \pi\right) .
$$

O termo exponencial na Equação (4) corresponde a parte radial do filtro e o outro termo é o componente angular do filtro. A função de espalhamento $\cos (d \theta)$ do componente angular varia entre -1 e 1 e, portanto, a adição do valor 1 seguida da divisão do resultado por 2 faz com que esse componente retorne valores no intervalo entre 0 e 1 . Os parâmetros $\omega_{s}, \theta_{n}$, $\sigma_{\beta}$ representam, respectivamente, o centro de frequência radial, o ângulo de orientação e a largura de banda radial. $N$ é o número de orientações dos filtros.

Na prática, a função de transferência de cada filtro, Equação (4), é multiplicada pela representação da imagem no domínio da frequência, $\mathcal{F}[I(x, y)]$, em que $\mathcal{F}$ indica a transformada de Fourier e, depois de transformar o resultado de volta para o domínio espacial aplicando a transformada de Fourier inversa, $\mathcal{F}^{-1}[\mathcal{F}[I(x, y)] \times G(\omega, \theta)]$, as partes real $\left(E_{s, n}\right)$ e imaginária $\left(O_{s, n}\right)$ do resultado complexo são usadas para calcular os componentes locais de energia $\left(A_{s, n}\right)$ e fase $\left(\phi_{s, n}\right)$ da imagem como:

$$
\begin{gathered}
A_{s, n}(\mathbf{x})=\sqrt{E_{s, n}^{2}(\mathbf{x})+O_{s, n}^{2}(\mathbf{x})}, \\
\phi_{s, n}(\mathbf{x})=\arctan \left(\frac{O_{s, n}(\mathbf{x})}{E_{s, n}(\mathbf{x})}\right) .
\end{gathered}
$$

2.2.3 Ajuste dos parâmetros da medida de congruência de fase Neste trabalho, os filtros de log-Gabor foram projetados para fornecer uma cobertura espectral uniforme, tendo 
Detecção de leucócitos em imagens de vídeo de microscopia intravital usando a técnica de congruência de fase

mínima sobreposição entre si e com respostas isotrópicas, visto que os leucócitos são estruturas simétricas e não apresentam orientação preferencial. Para que isso fosse alcançado, os valores dos parâmetros foram ajustados como: valor mínimo dos centros de frequência $\omega_{\min }=0,0135$ e número de escalas $S=4$, evitando assim problemas de aliasing em altas frequências e cobrindo uma faixa de frequências suficiente para mapear as variações de tamanho dos leucócitos; largura de banda radial $\sigma_{\beta}=0,59$; número de orientações dos filtros $N=4$, pois não há necessidade de realçar estruturas anisotrópicas. Neste estudo, para uma dada escala $s$, o centro de frequência radial $\left(\omega_{s}\right)$ do filtro foi calculado como $\omega_{s}=1 /\left(\omega_{\min } \times 2,1^{S-1}\right)$. O fator de ganho $\gamma$ e o valor de corte da sigmoide $c$ na Equação (2) foram fixados em 5 e 0,4 , respectivamente. Análises experimentais mostraram que os parâmetros $\gamma, c$ e $T$ possuem pouca influência sobre os resultados finais.

2.2.4 Matriz de momentos de PC A medida de PC, embora seja eficiente para a detecção de bordas em uma imagem, ignora a maneira como a congruência de fase varia em relação à orientação em cada ponto $\mathrm{x}$ da imagem. A fim de considerar a informação de orientação, Kovesi [14] propôs calcular o momento da medida PC para cada orientação e, então, analisar o comportamento dessas medidas. Para isso, foi proposto o uso de uma matriz de orientações de congruência de fase, que corresponde a uma matriz de momentos (ou covariância) da medida de PC $\left(M_{P C}\right)$. A análise dessa matriz de momentos é realizada a partir da sua decomposição em autovalores, os quais podem ser interpretados como o tamanho dos semieixos de uma elipse em 2D. Para cada pixel na posição espacial $\mathbf{x}$, a matriz de momentos é definida como:

$$
M_{P C}(\mathbf{x})=\left[\begin{array}{cc}
\sum_{n} P C_{x}^{2}(\mathbf{x}) & \sum_{n} P C_{x}(\mathbf{x}) P C_{y}(\mathbf{x}) \\
\sum_{n} P C_{x}(\mathbf{x}) P C_{y}(\mathbf{x}) & \sum_{n} P C_{y}^{2}(\mathbf{x})
\end{array}\right],
$$

em que, os valores de $P C_{x}(\mathbf{x})$ e $P C_{y}(\mathbf{x})$ correspondem, respectivamente, às projeções da medida PC nos eixos $x$ e $y$, para cada orientação $\phi_{n}$ do banco de filtros. Os momentos mínimo e máximo, equivalentes aos autovalores mínimo $\left(\lambda_{1}\right)$ e máximo $\left(\lambda_{2}\right)$ da matriz da Equação (8), são definidos por

$$
\lambda_{1,2}=\frac{1}{2}\left(c+a \mp \sqrt{b^{2}+(a-c)^{2}}\right),
$$

em que,

$$
\begin{gathered}
a=\sum_{n} P C_{x}^{2}(\mathbf{x}), \\
b=\sum_{n} P C_{x}(\mathbf{x}) P C_{y}(\mathbf{x}),
\end{gathered}
$$

$\mathrm{e}$

$$
c=\sum_{n} P C_{y}^{2}(\mathbf{x})
$$


Detecção de leucócitos em imagens de vídeo de microscopia intravital usando a técnica de congruência de fase

2.2.5 Medida de blobness Frangi et al. [8] desenvolveram uma função para o realce de estruturas tubulares em imagens que utiliza relações entre a magnitude dos autovalores da matriz Hessiana como um indicativo da forma de tais estruturas. Em Gregório da Silva et al. [10] tal função foi modificada para uma medida de blobness 2D que utiliza os autovalores da matriz Hessiana, formulada como

$$
B\left(\lambda_{1}, \lambda_{2}\right)=\left(1-\exp \left(-\frac{R_{A}^{2}}{2 \alpha^{2}}\right)\right) \times\left(1-\exp \left(-\frac{S^{2}}{2 \beta^{2}}\right)\right),
$$

em que, $\lambda_{1}$ e $\lambda_{2}$ correspondem aos autovalores da matriz Hessiana; $R_{A}=\lambda_{1} / \lambda_{2}$ é usado para minimizar a resposta da medida aos padrões em forma de linha e superfícies planas; $S=\sqrt{\lambda_{1}^{2}+\lambda_{2}^{2}}$ ajuda a reduzir a influência de pixels associados ao ruído na imagem, uma vez que eles apresentam autovalores muito baixos. Os parâmetros $\alpha$ e $\beta$, neste caso, podem ser ajustados para controlar a sensibilidade dos componentes do filtro.

Neste trabalho, a mesma medida de blobness da Equação (13) foi utilizada para o realce de estruturas circulares nas imagens de MI. A diferença é que, ao invés de utilizar os autovalores das matrizes Hessianas, foram utilizados os autovalores das matrizes de momentos de PC, em que, $0 \leq \lambda_{1} \leq \lambda_{2}$. Os valores da medida de blobness variam entre 0 e 1 , sendo que, quanto mais próximo de 1 , mais a estrutura local na imagem se aproxima da forma de uma bolha.

Para a obtenção dos parâmetros $\alpha$ e $\beta$ na Equação (13), primeiro variou-se o valor de $\alpha$ entre 0,1 a 0,9 (em passos de 0,1 ), mantendo o valor de $\beta$ fixo em 0,5 (valor proposto por Frangi et al. [8]). Em seguida, fixou-se o valor de $\alpha$ encontrado a partir do melhor resultado para a medida $\overline{\mathrm{AUCPR}}$ e o valor de $\beta$ foi variado de 0,1 a 0,9 (em passos de 0,1 ). Ao final, selecionou-se o valor de $\alpha=0,1$ e $\beta=0,7$ que produziu o máximo valor da $\overline{\mathrm{AUCPR}}$. Esses valores foram então utilizados para a obtenção dos resultados de detecção dos leucócitos.

2.2.6 Detecção dos leucócitos Após a geração do mapa da medida de blobness (contendo valores reais no intervalo entre 0 e 1 ), um algoritmo de busca por máximos locais 7 foi aplicado no mapa. Os máximos locais, possivelmente, correspondem aos leucócitos na imagem. $\mathrm{O}$ algoritmo foi aplicado com uma janela de $15 \times 15$ pixels, definida com base no tamanho médio dos leucócitos nas imagens de vídeo de MI, o valor do limiar $\left(T_{\text {pnax }}\right)$ que define o pixel como um máximo local foi variado entre 0,02 e 1,0 (em passos de 0,02 ). O número de máximos locais foi fixado em 40 , visto que esse valor é suficiente para capturar, com segurança, todos os leucócitos observados nos quadros do vídeo.

2.2.7 Casamento de padrões A técnica de casamento de padrões tenta encontrar os locais, em uma imagem $I(x, y)$, que correspondem a um determinado padrão $w(x, y)$ (imagem

${ }^{7}$ http://www.mathworks.com/help/vision/ref/vision.localmaximafinder-class.html 
Detecção de leucócitos em imagens de vídeo de microscopia intravital usando a técnica de congruência de fase

do objeto a ser detectado). Para uma imagem de tamanho $M \times N$, e um padrão de tamanho $J \times K$, em que $J \leq M$ e $K \leq N$, o pixel central do padrão é inicialmente posicionado sobre o pixel superior esquerdo da imagem e o coeficiente de Correlação Cruzada Normalizada (NCC) da Equação (14) é calculado. O padrão é então deslocado pixel a pixel sobre a imagem de busca até que o coeficiente de NCC seja calculado para todos os pixels, gerando uma imagem contendo os coeficientes de correlação que variam entre -1 e 1. Nesse caso, o valor 1 indica total correspondência entre o padrão e a imagem, 0 indica que não houve correspondência e - 1 indica que houve uma correspondência inversa (os valores de escala de cinza invertidos) entre o padrão e a imagem.

O NCC indica o grau de similaridade entre o padrão e a imagem de busca e é definido como

$$
\gamma(x, y)=\frac{\sum_{j}^{J} \sum_{k}^{K}[I(j, k)-\bar{I}(j, k)][w(x+j, y+k)-\bar{w}]}{\sqrt{\sum_{j}^{J} \sum_{k}^{K}[I(j, k)-\bar{I}(j, k)]^{2} \sum_{j}^{J} \sum_{k}^{K}[w(x+j, y+k)-\bar{w}]^{2}}},
$$

em que $\bar{w}$ é o valor médio dos pixels do padrão e $\bar{I}$ é o valor médio da imagem na região onde $I$ e $w$ se sobrepõem [9].

\subsection{Banco de imagens}

O vídeo de MI utilizado neste trabalho foi obtido de um experimento de MI da microvasculatura do cérebro de um camundongo em um estudo realizado no Núcleo de Neurociências da Universidade Federal de Minas Gerais (UFMG), sob a coordenação da Profa. Dra. Juliana Carvalho Tavares. O microscópio utilizado foi o Zeiss Imager M.2 (Thornwood, NY) com objetiva 20XLD. As características de aquisição das imagens do vídeo são: resolução espacial de 1,89 pixels $/ \mu \mathrm{m}$, matriz imagem de $420 \times 592$ pixels, 8 bits de quantização e taxa de amostragem de 16 quadros por segundo. O vídeo obtido possui um total de 220 quadros.

\subsection{Métricas de avaliação}

Os resultados da detecção de leucócitos foram avaliados utilizando as medidas de precisão, revocação e a medida - F1, que indicam a eficácia de um sistema de detecção [15]. Essas medidas são definidas como:

$$
\text { precisão }=\frac{\mathrm{TPs}}{\mathrm{TPs}+\mathrm{FPs}}
$$

e

$$
\text { revocação }=\frac{\text { TPs }}{\text { TPs }+ \text { FNs }},
$$


Detecção de leucócitos em imagens de vídeo de microscopia intravital usando a técnica de congruência de fase

em que, TPs (verdadeiros positivos) são os leucócitos corretamente detectados pela técnica proposta ao longo de todo o vídeo; FPs (falsos positivos) são os leucócitos detectados e que não correspondem aos leucócitos verdadeiros e FNs (falsos negativos) são os leucócitos que deixaram de ser detectados pela técnica. A medida-F1 [31] corresponde a média harmônica entre a precisão e a revocação e é definida como

$$
\text { medida-F1 }=2 \times \frac{\text { precisão } \times \text { revocação }}{\text { precisão }+ \text { revocação }} .
$$

Para as medidas definidas acima, quanto mais próximas elas estiverem do valor 1 , melhor será a eficácia do sistema. Os números de TPs, FPs e FNs foram determinados utilizando as localizações dos centroides dos leucócitos marcados manualmente (com o auxílio do plug-in "Manual Tracking" 8 do software ImageJ 9 , e as localizações dos pontos detectados pela técnica proposta nos quadros do vídeo de MI. Um ponto detectado foi considerado como TP se a distância Euclidiana entre ele e o centroide manualmente marcado mais próximo foi menor ou igual a 9 pixels. Esse valor de limiar (9 pixels) foi determinado com base no raio médio dos leucócitos presentes nas imagens. Um ponto detectado foi considerado como FP se a distância entre ele e qualquer outro ponto manualmente marcado foi superior a 9 pixels. Por fim, um ponto foi considerado como FN se este encontrava-se presente na lista de centroides manualmente marcados mas nenhuma correspondência foi detectada pela técnica proposta.

A avaliação do desempenho global do sistema foi realizada usando a metodologia de curvas de precisão-revocação e a média das áreas sob as curvas (Area Under the PrecisionRecall Curve - $\overline{\mathrm{AUCPR}}$ ), calculadas para cada quadro do vídeo processado. No caso, quanto mais próxima de 1 for a área, melhor será o desempenho do sistema.

\section{Resultados e discussões}

As duas técnicas usadas neste trabalho (técnica proposta e de casamento de padrões) foram analisadas da seguinte maneira. Primeiro, a detecção de leucócitos para ambas as técnicas foi avaliada para cada quadro do vídeo calculando-se os valores de TPs, FPs e FNs. Tais valores foram acumulados para todos os quadros permitindo o cálculo das métricas AUCPR

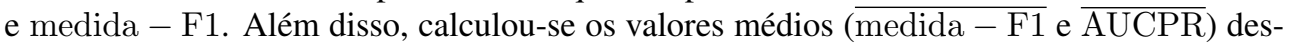
sas mesmas medidas a partir dos valores de contagem para cada quadro individualmente.

Para efeito de comparação, os resultados da técnica proposta por Gregório da Silva et al. [10], co-autor deste trabalho, são apresentados na terceira linha das Tabelas 1 e 2 Embora

\footnotetext{
${ }^{8}$ http://rsbweb.nih.gov/ij/plugins/track/track.html

${ }^{9}$ http://imagej.nih.gov/ij/
} 
Detecção de leucócitos em imagens de vídeo de microscopia intravital usando a técnica de congruência de fase

essa técnica utilize o mesmo procedimento para o pré-processamento das imagens, ela é baseada na análise dos autovalores da matriz Hessiana, ao invés da matriz de momentos usada pela técnica proposta neste trabalho, e a etapa de pós-processamento utiliza a informação do nível de intensidade de cada leucócito, ao invés da análise de máximos locais, conforme descrita na seção 2.2.6.

No caso da análise usando os valores de TPs, FPs e FNs acumulados, para a técnica proposta (medida de blobness) a medida - F1 foi igual a 0,790 , com valores de precisão e revocação iguais a 0,767 e 0,814 , respectivamente. Nesse caso, o valor do limiar $T_{p \max }$ utilizado foi igual a 0,16 . Para a técnica de casamento de padrões, a medida $-\mathrm{F} 1$ foi igual a 0,745 e os valores de precisão e revocação iguais a 0,756 e 0,735 , respectivamente. $\mathrm{O}$ valor de limiar $T_{\text {pmax }}$ utilizado neste caso foi igual a 0,64. Para a técnica de Hessiana [10], os valores de precisão, revocação e medida - F1 foram superiores aos da técnica de casamento de padrões. Em relação à técnica proposta, apenas o valor de precisão da técnica Hessiana foi superior. Os resultados para esse experimento são sumarizados na Tabela 1 .

Ainda em relação aos resultados apresentados na Tabela 1 , nota-se que o valor da AUCPR obtido para a técnica proposta foi superior às demais técnicas analisadas.

Tabela 1: Número de TPs, FPs e FNs e os valores das métricas precisão, revocação, medida - F1 e AUCPR obtidos com a técnica proposta, a técnica baseada na matriz Hessiana [10] e a de casamento de padrões. O número total de leucócitos presentes no vídeo é de 5588 .

\begin{tabular}{cccccccc}
\hline Técnica & TPs & FPs & FNs & precisão & revocação & medida-F1 & AUCPR \\
\hline técnica proposta & 4551 & 1380 & 1037 & 0,767 & 0,814 & 0,790 & 0,813 \\
casamento de padrões & 4108 & 1319 & 1480 & 0,756 & 0,735 & 0,745 & 0,733 \\
Hessiana [10] & 4227 & 1266 & 1361 & 0,770 & 0,760 & 0,760 & 0,700 \\
\hline
\end{tabular}

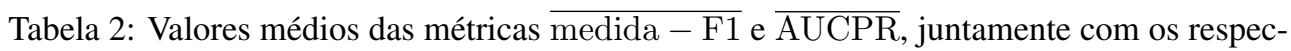
tivos intervalos de confiança, obtidos com a técnica proposta, a técnica baseada na matriz Hessiana [10] e a de casamento de padrões.

\begin{tabular}{ccc}
\hline Técnica & $\overline{\text { medida-F1 (IC: 95\%) }}$ & $\overline{\text { AUCPR }(I C: ~ 95 \%)}$ \\
\hline técnica proposta & $0,791[0,783,0,799]$ & $0,776[0,765,0,787]$ \\
casamento de padrões & $0,746[0,739,0,753]$ & $0,670[0,658,0,682]$ \\
Hessiana [10] & $0,787[0,778,0,796]$ & $0,670[0,660,0,690]$ \\
\hline
\end{tabular}

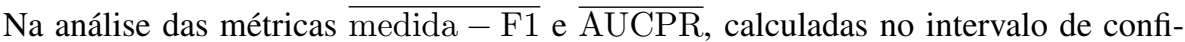
ança (IC) de 95\%, os valores obtidos para a técnica proposta foram, respectivamente, iguais a 0,791 e 0,776. Para este mesmo experimento, os valores obtidos para a técnica de casamento 
Detecção de leucócitos em imagens de vídeo de microscopia intravital usando a técnica de congruência de fase

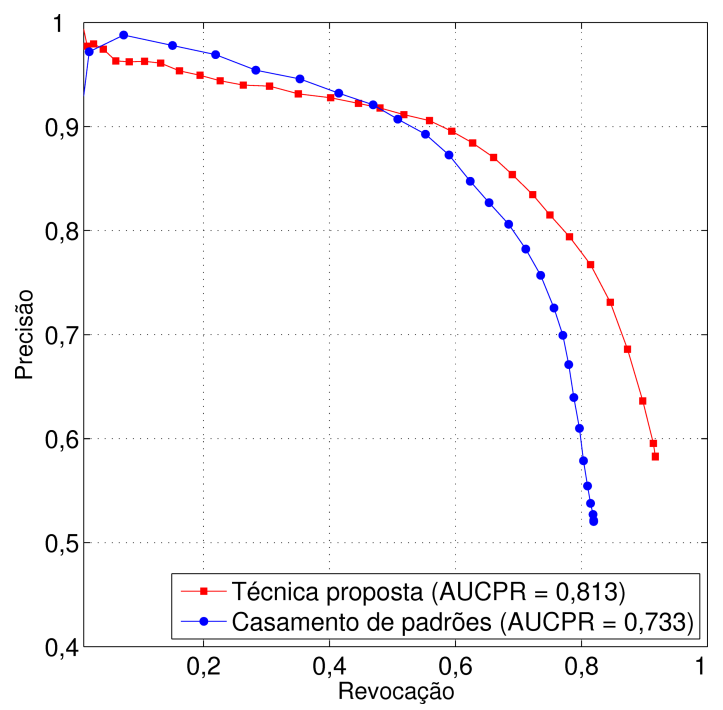

Figura 2: Curvas precisão-revocação obtidas para a técnica proposta (medida de blobness linha vermelha) e para a técnica casamento de padrões (linha azul) usando todos os quadros do vídeo de MI.

de padrões foram 0,746 e 0,670, respectivamente. Para a técnica Hessiana [10], apenas o valor da métrica medida - F1 foi significativamente maior que a técnica de casamento de padrões. Os resultados para esse experimento são sumarizados na Tabela 2. Com base nesses resulta-

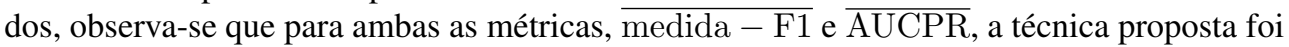
superior à técnica de casamento de padrões, levando-se em consideração o IC de 95\%.

Na Figura 2 são apresentadas as curvas de precisão-revocação resultantes da técnica proposta (medida de blobness - linha vermelha) e da técnica casamento de padrões (linha azul) obtidas usando todos os quadros do vídeo de MI. Cada ponto de operação dessas curvas foi obtido fixando-se o limiar $T_{\text {pmax }}$ para valores no intervalo de 0,02 a 1, em passos de 0,02. Analisando-se as curvas, nota-se que a técnica proposta apresentou um melhor desempenho global (AUCPR $=0,813)$ que a técnica de casamento de padrões (AUCPR $=0,733$ ).

Exemplos do processamento de um quadro do vídeo pela técnica proposta e pela técnica de casamento de padrões são apresentados na Figura 3 . O quadro original é apresentado na Figura 3 (a) em que os leucócitos marcados manualmente podem ser vistos como símbolos no formato " $\square$ " na Figura 3 (b). Na Figura 3 (c) e (d) os TPs são representados por círculos verdes, enquanto os círculos azuis e os símbolos " $\chi$ ” indicam os FPs e os FNs, respectivamente. Nessa imagem específica observa-se que a medida de blobness gerou um maior 
Detecção de leucócitos em imagens de vídeo de microscopia intravital usando a técnica de congruência de fase

número de TPs e um menor número de FPs e FNs que a técnica casamento de padrões.

É importante observar que embora as técnicas aqui propostas tenham sido desenvolvidas para a detecção de leucócitos em imagens de MI de camundongos, sua aplicação poderia ser facilmente estendida para outros experimentos (ratos ou humanos) que envolvam a detecção de células com características de contraste e forma semelhantes às analisadas neste trabalho.

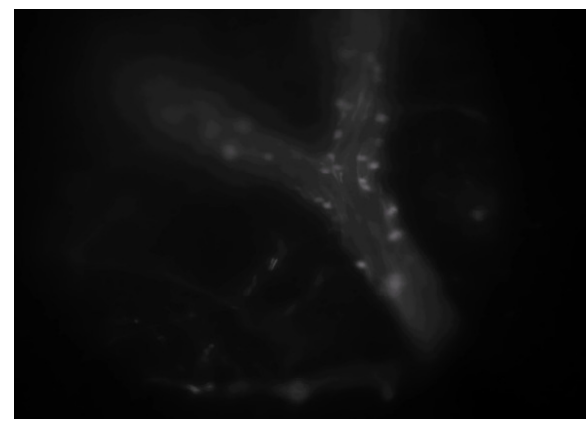

(a)

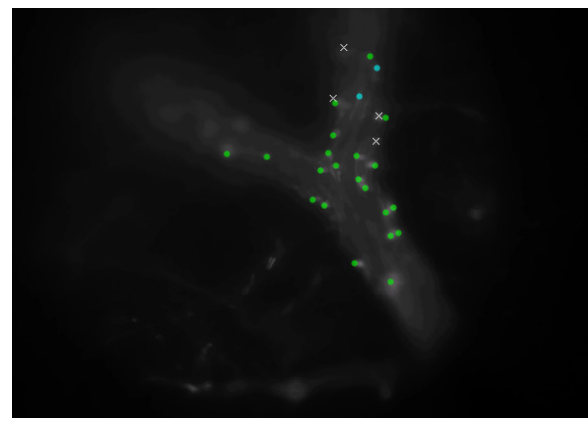

(c)

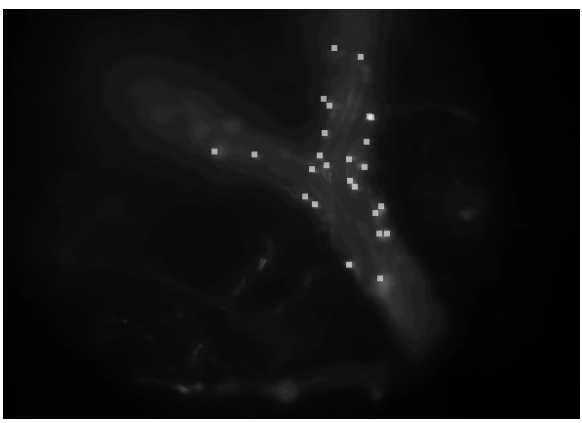

(b)

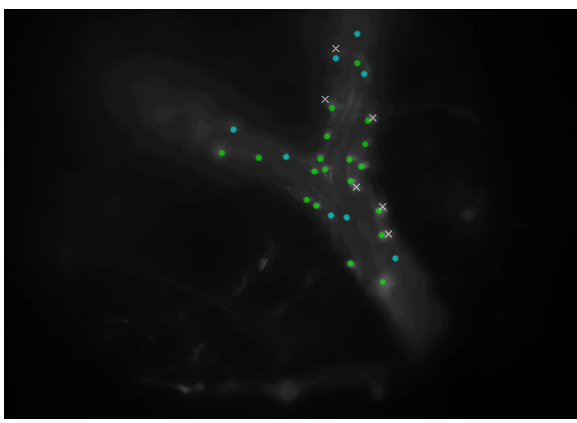

(d)

Figura 3: Resultado do processamento de um quadro do vídeo de MI. a) Quadro original; b) leucócitos marcados visualmente; c) resultado da técnica proposta; d) resultado da técnica casamento de padrões. Os círculos verdes indicam os TPs, os círculos azuis indicam os $F P s$ e os símbolos ' $\times$ ' indicam os FNs. 
Detecção de leucócitos em imagens de vídeo de microscopia intravital usando a técnica de congruência de fase

\section{Conclusões}

Neste trabalho foi proposta uma nova técnica de detecção automática de leucócitos em vídeos de MI. A medida de blobness proposta para realçar os leucócitos foi calculada com base na medida de PC, que possui como principal característica o fato de ser mais robusta a variações de iluminação e contraste nas imagens e, por isso, é menos suscetível ao efeito de Photobleaching. Os resultados da detecção foram avaliados usando a medida $-\mathrm{F} 1 \mathrm{e}$ a AUCPR e comparados com os das técnicas de casamento de padrões e matriz Hessiana [10]. Os resultados indicaram que a técnica proposta alcançou valores superiores a ambas as técnicas.

Como trabalho futuro, uma análise morfológica das células detectadas deverá ser realizada com o objetivo de reduzir o número de sinais falso positivos, ou seja, células erroneamente detectadas e identificadas como leucócitos.

\section{Agradecimentos}

Os autores agradecem à Coordenação de Aperfeiçoamento de Pessoal de Nível Superior (CAPES) e à Fundação de Amparo à Pesquisa do Estado de São Paulo (FAPESP processo $n^{\circ}$ 2013/26171-6) pelo apoio financeiro durante o desenvolvimento deste trabalho.

\section{Contribuição dos autores:}

Todos os autores tiveram contribuições aproximadamente iguais.

\section{Referências}

[1] S.T. Acton, K. Wethmar, and K. Ley. Automatic tracking of rolling leukocytes in vivo. Microvascular Research, 63(1):139-148, 2002.

[2] V. Andresen, K. Pollok, J-L. Rinnenthal, L. Oehme, R. Günther, H. Spiecker, H. Radbruch, J. Gerhard, A. Sporbert, Z. Cseresnyes, A.E. Hauser, and R. Niesner. Highresolution intravital microscopy. PLoS ONE, 7(12):e50915:1-12, 2012.

[3] J. Canny. A computational approach to edge detection. IEEE Transactions on Pattern Analysis and Machine Intelligence, 8(6):679-698, 1986.

[4] J. Cui, S.T. Acton, and Z. Lin. A Monte Carlo approach to rolling leukocyte tracking in vivo. Medical Image Analysis, 10(4):598-610, 2006. 
Detecção de leucócitos em imagens de vídeo de microscopia intravital usando a técnica de congruência de fase

[5] G. Dong, N. Ray, and S.T. Acton. Intravital leukocyte detection using the gradient inverse coefficient of variation. IEEE Transactions on Medical Imaging, 24(7):910924, 2005.

[6] M. Egmont-Petersen, U. Schreiner, S.C. Tromp, T.M. Lehmann, D.W. Slaaf, and T. Arts. Detection of leukocytes in contact with the vessel wall from in vivo microscope recordings using a neural network. IEEE Transactions on Biomedical Engineering, 47(7):941-951, 2000.

[7] R.J. Ferrari, C.H. Villa-Pinto, B.C. Gregório da Silva, D. Bernardes, and J. CarvalhoTavares. Automatic detection of motion blur in intravital video microscopy image sequences via directional statistics of log-gabor energy maps. Medical \& Biological Engineering \& Computing, 53(2):151-163, 2015.

[8] A.F. Frangi, W.J. Niessen, K.L. Vincken, and M.A. Viergever. Multiscale vessel enhancement filtering. In Medical Image Computing and Computer-Assisted Interventation MICCAI, volume 1496, pages 130-137, Cambridge, MA, USA, 1998. Springer-Verlag.

[9] R.C. Gonzalez, R.E. Woods, and S.L. Eddins. Digital Image Processing Using Matlab. Gatesmark Publishing, 2nd edition, 2009.

[10] B.C. Gregório da Silva, R.J. Ferrari, and J. Carvalho-Tavares. Detection of leukocytes in intravital video microscopy based on the analysis of hessian matrix eigenvalues. In Conference on Graphics, Patterns and Images (SIBGRAPI), pages 345-352, Salvador, Bahia, 2015.

[11] B.C. Gregório da Silva, P.G. Freire, D. Bernardes, J. Carvalho-Tavares, R.F. Mello, and R.J. Ferrari. Técnica de estabilização de movimento em microscopia intravital utilizando métodos de co-registro de imagens. In XXIV Congresso Brasileiro de Engenharia Biomédica, pages 193-196, 2014.

[12] P. Kovesi. Invariant measures of image features from phase information. $\mathrm{PhD}$ thesis, University of Western Australia, Perth, Western, Australia, 1996.

[13] P. Kovesi. Image features from phase congruency. Videre: Journal of computer vision research, 1(3):1-26, 1999.

[14] P. Kovesi. Phase congruency detects corners and edges. In The australian pattern recognition society conference: DICTA 2003, pages 309-318, Sydney, New South Wales, Australia, 2003.

[15] T.C. Landgrebe, P. Paclik, R.P.W. Duin, and A.P. Bradley. Precision-recall operating characteristic (P-ROC) curves in imprecise environments. In 18th International Conference on Pattern Recognition - ICPR, volume 4, pages 123-127, 2006. 
Detecção de leucócitos em imagens de vídeo de microscopia intravital usando a técnica de congruência de fase

[16] D.G. Lowe. Distinctive image features from scale-invariant keypoints. International Journal of Computer Vision, 60(2):91-110, 2004.

[17] D. Marr and E. Hildreth. Theory of edge detection. Proceedings of the Royal Society B: Biological Sciences, 207(1167):187-217, 1980.

[18] A. Masedunskas, O. Milberg, N. Porat-Shliom, M. Sramkova, T. Wigand, P. Amornphimoltham, and R. Weigert. Intravital microscopy: A practical guide on imaging intracellular structures in live animals. Bioarchitecture, 2(5):143-157, 2012.

[19] G. Medioni, S. Menet, and P. Saint-Marc. B-Snakes: implementation and application to stereo. In Proceedings of the 7th Israeli Conference on Artificial Intelligence, Vision and Pattern Recognition, pages 223-236, Tel-Aviv, Israel, 1990.

[20] M.C. Morrone and D.C. Burr. Feature detection in human vision: A phase-dependent energy model. In Proceedings of the Royal Society of London, volume 235 of Series B, Biological Sciences, pages 221-245, 1988.

[21] M.C. Morrone and R.A. Owens. Feature detection from local energy. Pattern Recognition Letters, 6(5):303-313, 1987.

[22] N.H. Nguyen, S. Keller, E. Norris, T.T. Huynh, M.G. Clemens, and M.C. Shin. Tracking colliding cells in vivo microscopy. IEEE Transactions on Biomedical Engineering, 58(8):2391-2400, 2011.

[23] L.G. Nyúl, J.K. Udupa, and X. Zhang. New variants of a method of MRI scale standardization. IEEE Transactions on Medical Imaging, 19(2):143-150, 2000.

[24] V. Pinho, F.M. Coelho, G.B. Menezes, and D.C. Cara. Intravital microscopy to study leukocyte recruitment in vivo. In Light Microscopy - Methods in Molecular Biology, volume 689, chapter 6, pages 81-90. Humana Press, USA, 2011.

[25] M.J. Pittet and R. Weissleder. Intravital imaging. Cell, 147(5):983-991, 2011.

[26] N. Ray, S.T. Acton, and K. Ley. Tracking leukocytes in vivo with shape and size constrained active contours. IEEE Transactions on Medical Imaging, 21(10):1222-1235, 2002 .

[27] Y. Sato, J. Chen, R. Zoroofi, N. Harada, S. Tamura, and T. Shiga. Automatic extraction and measurement of leukocyte motion in microvessels using spatiotemporal image analysis. IEEE Transactions on Biomedical Engineering, 44(4):225-236, 1997.

[28] C. Sumen, T.R. Mempel, I.B. Mazo, and U.H. Andrian. Intravital microscopy: Visualizing immunity in context. Immunity, 21(3):315-329, 2004. 
Detecção de leucócitos em imagens de vídeo de microscopia intravital usando a técnica de congruência de fase

[29] C. Tomasi and R. Manduchi. Bilateral filtering for gray and color images. In Sixth International Conference on Computer Vision, pages 839-846, Bombay, India, 1998.

[30] Q. Wu, F. Merchant, and K. Castleman. Microscope image processing. Academic Press, San Diego, California, USA, 2010.

[31] E. Zhang and Y. Zhang. F-measure. In L. Liu and M.T. Özsu, editors, Encyclopedia of Database Systems, page 1147. Springer US, New York, USA, 2009. 